\title{
Prevalence of sleep disturbance and the association between poor disease control in people with ankylosing spondylitis within the Australian clinical setting (ASLEEP study): a real-world observational study using the OPAL dataset
}

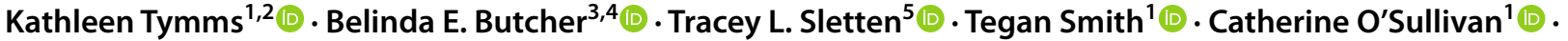

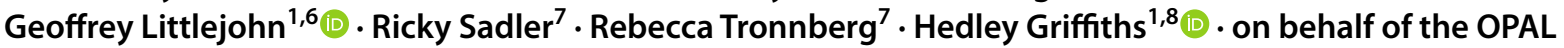 \\ consortium
}

Received: 8 July 2021 / Revised: 27 September 2021 / Accepted: 30 September 2021 / Published online: 26 November 2021

(c) International League of Associations for Rheumatology (ILAR) 2021

\begin{abstract}
Introduction Sleep disturbance and fatigue are commonly reported in ankylosing spondylitis (AS) but specific prevalence and the relationship to disease control are unknown.

Method This retrospective non-interventional observational study of data from the OPAL dataset included patients with AS (ICD code M45, M45.0 or M08.1), aged 18 to 95 years and had completed $\geq 1$ sleep questionnaire between 1 January 2019 and 30 September 2020. The prevalence of insomnia and obstructive sleep apnoea were assessed using the Insomnia Severity Index (ISI) and Multivariate Apnoea Prediction Index (MAPI), respectively. Propensity score (PS) matching based on sex, age and symptom duration increased comparability between patients administered tumour necrosis factor inhibitors (TNFi) and interleukin 17A inhibitors (IL-17Ai).

Results Four hundred ninety-five patients were included. The mean ISI total score in the overall population was 8.6 \pm 6.2. Self-reported moderate or severe clinical insomnia was present in $16 \%$ and $3.2 \%$ of patients, respectively. The mean MAPI score was $0.4 \pm 0.3$, self-reported apnoea was identified in $31.5 \%$ of patients and the mean FACIT-Fatigue score was $36.1 \pm 10.7$. In the PS matched population, the only treatment-related difference was the mean MAPI score (IL-17Ai $0.4 \pm 0.3$ and TNFi $0.3 \pm 0.2, p=0.046$ ). Those with poor disease control (BASDAI $\geq 4$ ) were more likely (odds ratio [OR] $7.29,95 \%$ CI 2.37 to $22.46, p=0.001$ ) to have a greater severity of insomnia symptoms than those with good disease control. Conclusion In this real-world AS cohort, poor disease control was associated with sleep disturbance. Little difference in sleep disturbance was observed between biologic TNFi and IL-17Ai treatment.
\end{abstract}

\section{Key Points}

- Sleep disturbance and fatigue are common in patients with ankylosing spondylitis.

- In our real-world cohort, self-reported apnoea was reported in one-third of patients; and one in five patients reported moderate to severe insomnia.

- Those with poor disease control were more likely to experience greater sleep disturbance than those with good disease control.

Kathleen Tymms

KTymms@canberrarheumatology.com.au

1 OPAL Rheumatology Ltd, Sydney, NSW, Australia

2 Canberra Rheumatology, 9/40 Marcus Clarke St, Canberra, ACT 2601, Australia

3 University of New South Wales, Kensington, NSW, Australia

4 WriteSource Medical Pty Ltd, Lane Cove, NSW, Australia
5 Turner Institute for Brain and Mental Health, School of Psychological Sciences, Monash University, Clayton, VIC, Australia

6 Department of Medicine, Monash University, Clayton, VIC, Australia

7 Novartis Pharmaceuticals Australia Pty Ltd, Macquarie Park, NSW, Australia

8 Barwon Rheumatology Service, Geelong, VIC, Australia 
Keywords Ankylosing spondylitis · Insomnia $\cdot$ Interleukin 17A inhibitors $\cdot$ Sleep apnoea $\cdot$ Sleep disorders $\cdot$ Tumour necrosis factor inhibitors

\section{Introduction}

Ankylosing spondylitis (AS) is characterized by inflammation of entheseal structures and the adjacent bone marrow resulting in structural damage and new bone formation in spine, sacroiliac joints and other entheseal sites. As the control of inflammatory back pain and stiffness improves with the introduction of biologic disease modifying antirheumatic drugs (bDMARDs), clinicians are now turning their attention to comorbidities and extra-articular manifestations of AS, including sleep disturbances and fatigue. Sleep quality and fatigue are important contributors to overall quality of life [1].

A sleep disorder is a condition that interferes with the quality, timing and duration of sleep. The most common types of sleep disorders include insomnia, sleep-related breathing disorders such as sleep apnoea, circadian rhythm disorders and sleep movement disorders. Others have reported that sleep disorders (including difficulties with sleep quality, sleep latency, sleep efficiency and sleep medication use) are more prevalent in AS patients compared to the general population, particularly in AS patients with high disease activity [1]. Sleep disturbance and fatigue are common features of AS [2,3] with sleep disturbance prevalence estimates varying between 59 and $90 \%$ [1, 3, 4]. Sleep disturbances are associated with increased pain, fatigue, disease activity, mood and depressive symptoms [1, 4].

The causes of sleep disturbance in patients with AS are not fully understood and are likely to be multifactorial. For example, pathological changes caused by AS including spinal deformities may cause pain, and thoracic manifestations affecting the chest wall, airways, lung parenchyma, heart and great vessels with cervical spine deformities can affect upper airways, for example via limited thoracic expansion [5]. Similarly, depression which has a higher prevalence in patients with AS influences sleep disturbance, although there is a bidirectional relationship with depression, so it is difficult to understand which is causal [6]. The pro-inflammatory cytokines (TNF $\alpha$ and IL-1) have been implicated in sleep regulation, enhancing non-rapid eye movement sleep (NREM) and inducing sleepiness [7]. There is also an association between AS and obstructive sleep apnoea, which in turn is linked with excessive daytime sleepiness, hypertension, cardiovascular disease and fatigue [2]. Interestingly, TNF $\alpha$ is elevated in patients with sleep apnoea (in the absence of any concomitant rheumatological diagnosis) [8], and continuous positive airway pressure has been shown to decrease soluble TNF in this setting [9]. There is some evidence to suggest that the frequency of obstructive sleep apnoea is lower in patients receiving tumour necrosis factor inhibitors (TNFi) compared to those not taking TNFi, although this study was small [10].

While there have been some cross-sectional studies conducted elsewhere [5], to date, there has not been studies performed to assess the prevalence of sleep disturbances in a real-world cohort of patients with AS in Australia. To address this gap, the primary objective of this study is to estimate the prevalence of sleep disturbances in patients with AS who are included in the OPAL (Optimizing Patient outcomes in Australian RheumatoLogy) dataset. The secondary objectives included the impact of therapy for AS on sleep outcomes and correlations between disease activity and fatigue with sleep disturbance.

\section{Method}

\section{Study design}

This is a cross-sectional study of observational data embedded within the OPAL dataset. The OPAL dataset contains de-identified information captured during routine clinical care using purpose-built worksheets in the Audit4 electronic medical record (EMR) [11, 12]. A protocol for this study was developed and approved by the institutional ethics committee prior to analysis. The protocol was not registered on the Open Science Framework, nor was it published. A statistical analysis plan was also developed and approved prior to analysis. No post hoc changes to the statistical analysis plan were made.

Data from patients with a physician diagnosis of AS (ICD code M45, M45.0 or M08.1) in the OPAL dataset, were alive at the time of the data cut and between 18 and 95 years and had completed at least one Insomnia Severity Index (ISI) or Multivariate Apnoea Prediction Index (MAPI) questionnaire between 1 January 2019 and 30 September 2020 were included in the study. Variables used in this study included the patient's demographics (sex, age, body mass index, symptom duration [defined as the date of sleep questionnaire administration minus the date of onset of symptoms], line of biologic therapy), disease severity (Bath Ankylosing Spondylitis Disease Activity Index [BASDAI], erythrocyte sedimentation rate [ESR], c-reactive protein [CRP]), treatment (corticosteroid, NSAID, bDMARD), the presence of co-morbid depression (ICD codes F06.32, F10.8, F11.8, F13.8, F14.8, F15.8, F16.8, F18.8, F19.8, F32, F33 or F34.1) and responses to patient-reported outcome questionnaires on sleep (ISI, MAPI, Functional Assessment of Chronic Illness Therapy-Fatigue [FACIT-Fatigue]). Follow-up information was collected until 30 September 2020. 
The activities of OPAL Rheumatology Ltd have received overarching ethics approval from the University of NSW Human Research Ethics Committee (UNSW HREC), based on a patient opt-out arrangement (HC17799). This research protocol was approved by the UNSW HREC (HC180712).

\section{Patient-reported outcomes (PROs)}

Validated PROs (ISI, MAPI, FACIT-Fatigue) were sent from the patients EMR within Audit4 and delivered to the patient's email address at time intervals specified by their treating rheumatologist or completed in the clinic waiting room using a tablet or the patient's smart phone. Completed questionnaires were encrypted and returned to the patient's Audit4 EMR for review at the next consultation [12].

\section{Objectives and outcomes}

The primary objective was to determine the prevalence of sleep disorders as measured by the number of patients with AS who were identified as being at risk of insomnia or obstructive sleep apnoea using the ISI or MAPI during the index period divided by the total number of patients with AS who had completed a survey.

The secondary objectives were to assess the relationship between demographic factors and sleep disturbance; to assess the impact of therapy for AS on sleep outcomes; to assess the prevalence of sleep disturbance in patients by AS therapy, specifically treatment with a tumour necrosis factor inhibitor (TNFi) or an interleukin 17A inhibitor (IL-17Ai), at index; and to assess the correlation between clinical signs and symptoms (measured by the BASDAI and FACIT-Fatigue), with sleep disturbance.

\section{Statistical methods}

All patients with clinical data in the OPAL dataset that met the inclusion/exclusion criteria were included in the study. Assuming that 500 patients completed the questionnaires (of an approximate patient population with AS in the OPAL dataset of 3,800), then if the prevalence of sleep disturbance was $65 \%$ in either treatment group (based on previously published data [13]), the confidence interval around IL-17Ai users was calculated to be 51.2 to $78.8 \%$ and around TNFi users was calculated to be 60.5 to $69.5 \%$. This assumes that IL-17Ai users made up $10 \%$ of the PRO completers, and TNFi users made up $90 \%$ of the PRO completers. The proportions of patients prescribed IL-17Ai vs TNFi was based analysis of prescribing patterns from the OPAL dataset.

Due to the potential biases in observational data, secondary analyses used a propensity score matching method to increase the comparability of the observed index characteristics of patients treated with IL-17Ai compared to TNFi. The propensity score is the conditional probability of receiving treatment (that is IL-17Ai vs TNFi) which was estimated using linear regression. We planned to use age group at index, sex, BASDAI score, duration of disease, concomitant therapy and line of therapy as covariates in the model. We planned to use a ratio of one patient prescribed IL-17Ai to every ten patients prescribed TNFi, which reflected the current treatment usage patterns at the time of study initiation. We assessed the success of matching by comparing the standardized differences in means and proportions between groups, with differences of more than 0.1 considered substantial differences [14]. We were unable to find suitable matches on all the planned covariates due to missing data or inadequate matching; therefore, the propensity score matching was conducted using sex, age category and disease duration. BASDAI category was missing for $183(37 \%)$ of patients, and suitable matches on concomitant medication and line of therapy were not possible. We used a ratio of one user of IL-17Ai to every two users of TNFi. Further matching was not possible due to inadequate matches being available within the dataset.

Patients were defined as having no clinically significant insomnia (ISI score $\leq 7$ ), subthreshold insomnia (ISI $>7$ and $\leq 14$ ), moderate severity clinical insomnia (ISI score $>14$ and $\leq 21$ ) or severe clinical insomnia (ISI score $>21$ ) [15]. Patients were considered to have poor disease control if their BASDAI score was $\geq 4$ [16]. The MAPI is a score for predicting sleep apnoea in patients [17]. Scores range from 0 to 1 , with higher scores associated with an increased probability of sleep apnoea. MAPI values higher than 0.5 are suggestive of clinical apnoea [17].

The association of demographic factors on sleep disturbance (ISI category, MAPI) was assessed using ordinal or logistic regression (as appropriate). Covariates included in the model were age (category), sex, weight, body mass index (BMI), symptom duration, BASDAI score $(<4$ vs $\geq 4)$ and use of steroids (no vs yes), non-steroidal anti-inflammatory drugs (NSAIDs; no vs yes) and bDMARDs (IL-17Ai vs TNFi).

All analyses were conducted in Stata MP V16.1 for Mac (StataCorp, Texas Station, USA). Missing data were not imputed. All tests were conducted two sided and values of $p<0.05$ were considered statistically significant.

\section{Results}

\section{Participants}

Of the 5,323 patients with AS in the OPAL dataset, 629 (11.8\%) were sent sleep questionnaires and 495 (9.3\%) were included in the study ( $79 \%$ response rate), and the demographics are reported in Table 1. Of the 495 
Table 1 Patient demographics

\begin{tabular}{|c|c|c|}
\hline Factor & Level & Value \\
\hline$N$ & & 495 \\
\hline Age at index (years), mean (SD) & & $48.3(13.6)(n=495)$ \\
\hline \multirow[t]{6}{*}{ Age category (years) } & 18-34 years & $79(16.0 \%)$ \\
\hline & $35-44$ years & $117(23.6 \%)$ \\
\hline & $45-54$ years & $138(27.9 \%)$ \\
\hline & $55-64$ years & $103(20.8 \%)$ \\
\hline & $65-74$ years & $40(8.1 \%)$ \\
\hline & $75-95$ years & $18(3.6 \%)$ \\
\hline \multirow[t]{3}{*}{ Sex } & Male & $274(55.4 \%)$ \\
\hline & Female & $220(44.4 \%)$ \\
\hline & Missing & $1(0.2 \%)$ \\
\hline \multirow[t]{5}{*}{ BMI category } & Underweight & $8(1.6 \%)$ \\
\hline & Normal weight & $142(28.7 \%)$ \\
\hline & Overweight & $144(29.1 \%)$ \\
\hline & Obese & $168(33.9 \%)$ \\
\hline & Missing & $33(6.7 \%)$ \\
\hline Symptom duration (months), mean (SD) & & $164.4(151.4)(n=495)$ \\
\hline \multirow[t]{7}{*}{ Line of bDMARD therapy } & 1 & $371(74.9 \%)$ \\
\hline & 2 & $51(10.3 \%)$ \\
\hline & 3 & $17(3.4 \%)$ \\
\hline & 4 & $5(1.0 \%)$ \\
\hline & 5 & $1(0.2 \%)$ \\
\hline & 7 & $1(0.2 \%)$ \\
\hline & No prior bDMARD & $49(9.9 \%)$ \\
\hline Duration of treatment (months), mean (SD) & & $57.9(107.3)(n=311)$ \\
\hline \multirow[t]{3}{*}{ BASDAI category } & Optimal disease control $(<4)$ & $256(51.7 \%)$ \\
\hline & Suboptimal disease control & $56(11.3 \%)$ \\
\hline & Missing & $183(37.0 \%)$ \\
\hline ESR, median (IQR) & & $6.0(3.0,14.0)(n=399)$ \\
\hline CRP, median (IQR) & & $2.8(1.0,4.2)(n=409)$ \\
\hline \multirow[t]{2}{*}{ Depression } & No & $471(95.2 \%)$ \\
\hline & Yes & $24(4.8 \%)$ \\
\hline
\end{tabular}

BASDAI, Bath Ankylosing Spondylitis Disease Activity Index; $B D M A R D$, biological disease modifying antirheumatic drug; $B M I$, body mass index; $I Q R$, interquartile range; $S D$, standard deviation

patients, 48 (9.7\%) were receiving IL-17Ai, 395 (79.8\%) were receiving TNFi and $52(10.5 \%)$ were receiving other biological and non-biological treatments. For patients receiving $\mathrm{TNFi}$, the majority were receiving adalimumab $(n=167 / 395,42.3 \%)$ or etanercept $(n=92 / 395,23.3 \%)$. A greater proportion of women were receiving IL-17Ai inhibitors compared to TNFi or other treatments $(64.6 \%$, $41.8 \%$ and $46.2 \%$, respectively, $p<0.001$ ), and less patients in the IL-17Ai group were receiving the drug as a first line biologic treatment compared to TNFi (64.6\% vs $85.3 \%, p=0.01)$. Median CRP was lower in the IL-17Ai group compared to other groups $(2.0$ in IL-17Ai compared to 3.0 in TNFi or 3.4 in other treatment, $p=0.026$ ).
There were no other clinically relevant differences in index demographics in the overall population.

In the propensity score matched set, 48 patients were prescribed IL-17Ai at index, and 94 were prescribed TNFi at index (Table 2). Patients in each group were well matched, with the exception of line of therapy, where there was a higher proportion of TNFi users in first line of bDMARD therapy compared to IL-17Ai users (82\% vs $65 \%, p=0.034)$.

\section{Proportion of patients with sleep disorders at index}

At index, the mean ISI total score in the overall population was $8.6 \pm 6.2$. Moderate or severe clinical insomnia 
Table 2 Patient demographics (propensity score matched population)

\begin{tabular}{|c|c|c|c|c|}
\hline Factor & Level & TNFi & IL-17Ai & $p$ value \\
\hline$N$ & & 94 & 48 & \\
\hline Age at index (years), mean (SD) & & $48.8(12.8)(n=94)$ & $51.1(14.7)(n=48)$ & 0.34 \\
\hline Age at index (years), median (IQR) & & $47.0(40.0,58.0)(n=94)$ & $48.0(40.5,61.5)(n=48)$ & 0.46 \\
\hline \multirow[t]{6}{*}{ Age category (years) } & 18-34 years & $11(12 \%)$ & $5(10 \%)$ & \multirow[t]{6}{*}{0.47} \\
\hline & $35-44$ years & $21(22 \%)$ & $12(25 \%)$ & \\
\hline & $45-54$ years & $34(36 \%)$ & $13(27 \%)$ & \\
\hline & $55-64$ years & $13(14 \%)$ & $9(19 \%)$ & \\
\hline & $65-74$ years & $14(15 \%)$ & $6(12 \%)$ & \\
\hline & 75-95 years & $1(1 \%)$ & $3(6 \%)$ & \\
\hline \multirow[t]{3}{*}{ Sex } & Male & $30(32 \%)$ & $16(33 \%)$ & \multirow[t]{3}{*}{0.36} \\
\hline & Female & $64(68 \%)$ & $31(65 \%)$ & \\
\hline & Missing & $0(0 \%)$ & $1(2 \%)$ & \\
\hline BMI (kg/m^2), mean (SD) & & $30.7(26.7)(n=86)$ & $31.0(8.9)(n=46)$ & 0.94 \\
\hline \multirow[t]{5}{*}{ BMI category } & Underweight & $2(2 \%)$ & $1(2 \%)$ & \multirow[t]{5}{*}{0.094} \\
\hline & Normal weight & $33(35 \%)$ & $8(17 \%)$ & \\
\hline & Overweight & $24(26 \%)$ & $16(33 \%)$ & \\
\hline & Obese & $27(29 \%)$ & $21(44 \%)$ & \\
\hline & Missing & $8(9 \%)$ & $2(4 \%)$ & \\
\hline Symptom duration (months), mean (SD) & & $127.0(116.5)(n=94)$ & $143.0(149.8)(n=48)$ & 0.49 \\
\hline Symptom duration (months), median (IQR) & & $96.3(35.9,182.6)(n=94)$ & $75.7(26.6,249.6)(n=48)$ & 0.98 \\
\hline \multirow[t]{5}{*}{ Line of bDMARD therapy } & 1 & $77(82 \%)$ & $31(65 \%)$ & \multirow[t]{5}{*}{0.034} \\
\hline & 2 & $8(9 \%)$ & $12(25 \%)$ & \\
\hline & 3 & $7(7 \%)$ & $4(8 \%)$ & \\
\hline & 4 & $2(2 \%)$ & $0(0 \%)$ & \\
\hline & 5 & $0(0 \%)$ & $1(2 \%)$ & \\
\hline Duration of treatment (months), mean (SD) & & $61.0(156.7)(n=69)$ & $23.7(20.1)(n=32)$ & 0.18 \\
\hline \multirow[t]{3}{*}{ BASDAI category } & Optimal disease control $(<4)$ & $43(46 \%)$ & $23(48 \%)$ & \multirow[t]{3}{*}{0.89} \\
\hline & Suboptimal disease control & $16(17 \%)$ & $8(17 \%)$ & \\
\hline & Missing & $35(37 \%)$ & $17(35 \%)$ & \\
\hline ESR, mean (SD) & & $12.0(13.5)(n=81)$ & $10.2(8.8)(n=41)$ & 0.43 \\
\hline ESR, median (IQR) & & $8.0(4.0,15.0)(n=81)$ & $8.0(4.0,14.0)(n=41)$ & 0.68 \\
\hline CRP, mean (SD) & & $4.7(7.3)(n=81)$ & $5.6(6.9)(n=41)$ & 0.55 \\
\hline CRP, median (IQR) & & $2.5(1.0,4.2)(n=81)$ & $3.0(2.0,6.0)(n=41)$ & 0.15 \\
\hline \multirow[t]{2}{*}{ Depression } & No & $90(96 \%)$ & $45(94 \%)$ & \multirow[t]{2}{*}{0.60} \\
\hline & Yes & $4(4 \%)$ & $3(6 \%)$ & \\
\hline
\end{tabular}

BASDAI, Bath Ankylosing Spondylitis Disease Activity Index; bDMARD, biological disease modifying antirheumatic drug; BMI, body mass index; $I Q R$, interquartile range; $S D$, standard deviation

based on the ISI was present in $16 \%$ and $3.2 \%$ of patients, respectively. This was largely driven by reported difficulties staying asleep, dissatisfaction with sleep and sleep interference. The mean MAPI score at index was $0.4 \pm 0.3$, with $31.5 \%$ of evaluable patients (that is patients with a non-missing MAPI questionnaire) identified as being at risk of sleep apnoea. Few patients self-reported stopping breathing (rarely $10.9 \%$, sometimes $5.1 \%$, frequently $1.6 \%$ or always $1.6 \%$ ). Moderate or severe clinical insomnia based on the ISI and a risk of sleep apnoea based on MAPI score $>0.5$ was reported in $5.6 \%$ of patients. The mean
FACIT-Fatigue total score at index was $36.1 \pm 10.7$. The greatest difficulties reported in FACIT-Fatigue subscales were with fatigue, tiredness, energy levels and interference with usual activities.

\section{Associations between therapy and sleep disturbance}

In the overall population, there were statistically significant differences in the proportion of patients reporting the following symptoms on the ISI: difficulty staying asleep 
(severe or very severe problems: IL-17Ai $14.6 \%$, TNFi $9.6 \%$, other $26.9 \%, p=0.002$ ); satisfaction with their sleep (dissatisfied or very dissatisfied: IL-17Ai $20.8 \%$, TNFi $23.5 \%$, other $40.4 \%, p=0.011$ ); experiencing sleep interference (much or very much: IL-17Ai $18.7 \%$, TNFi $14.7 \%$, other $27.2 \%, p<0.001$ ); problems being noticeable to others (much or very much: IL-17Ai $12.5 \%$, TNFi $8.8 \%$, other $21.1 \%, p=0.007$ ); and worry or distress (much or very much: IL-17Ai 10.4\%, TNFi 9.9\%, other $12.4 \%, p=0.012$ ). However, there was no difference between the groups in the proportion of patients with clinically suspected moderate or severe insomnia (IL-17Ai $20.9 \%$, TNFi $16.0 \%$, other $28.9 \%$, $p=0.079$ ).

There were some treatment-related differences in the propensity score matched population: the mean MAPI index was higher in those with IL-17Ai at index (IL-17Ai 0.4 \pm 0.3 and TNFi $0.3 \pm 0.2, p=0.046$ ), and the proportion at risk of sleep apnoea based on the MAPI score was higher in those treated with IL-17Ai than those treated with TNFi $(66 \%$ vs $54 \%, p=0.051$ ), although this did not reach statistical significance.

\section{Association between demographic factors on sleep disturbance}

In the propensity score matched population, those with poor disease control (BASDAI $\geq 4$ ) were seven times more likely (odds ratio [OR] 7.29, 95\% CI 2.37 to $22.46, p=0.001$ ) to have a greater severity of insomnia symptoms as measured by ISI category than those with good disease control. Those taking steroids at index were almost four times more likely (OR 3.98, 95\% CI 1.03 to $15.34, p=0.045$ ) to experience greater sleep disturbance than those not taking steroids. There were 87 patients included in this model. No other factors were significant (Table 3 ). It should be noted that model fit was relatively poor (McFadden's pseudo $R$-squared 0.2516 ), the model is likely over specified (that is the number of covariates in the model is not supported by the number of patients included in it), and there were wide confidence intervals.

In the MAPI model, females were less likely (OR 0.00, $95 \%$ CI 0.00 to $0.13, p<0.001$ ) to be at risk of sleep apnoea; those reported as taking NSAIDs within the EMR were 12 times more likely to be at risk of sleep apnoea (OR 12.1, 95\% CI 1.42 to $103.1, p=0.022$ ) compared to those not taking NSAIDs. No other factors were significant. Again, model fit was poor (McFadden's pseudo $R$-squared 0.5518 ), and for some factors there are extremely wide confidence intervals, and the model is likely over specified (that is the number of covariates in the model is not supported by the number of patients within the model as it included only 83 patients).
Table 3 Relationship between ISI category and demographic factors in the propensity score matched population, ordinal regression $(n=87)$

\begin{tabular}{|c|c|c|c|}
\hline Demographic factor & OR & $95 \%$ CI & $p$ value \\
\hline \multicolumn{4}{|l|}{ Age } \\
\hline 18-34 years & Reference & & \\
\hline 35-44 years & 0.24 & $0.03-1.87$ & 0.172 \\
\hline $45-54$ years & 0.39 & $0.06-2.43$ & 0.313 \\
\hline $55-64$ years & 0.05 & $0.01-0.54$ & 0.013 \\
\hline $65-74$ years & 0.25 & $0.03-2.00$ & 0.193 \\
\hline 75-94 years & $1.06 \times 10^{-7}$ & & 0.990 \\
\hline \multicolumn{4}{|l|}{ Sex } \\
\hline Male & Reference & & \\
\hline Female & 1.86 & $0.53-6.51$ & 0.332 \\
\hline Other & (Omitted) & & \\
\hline \multicolumn{4}{|l|}{ BMI } \\
\hline Underweight & Reference & & \\
\hline Normal weight & 2.09 & $0.08-55.06$ & 0.658 \\
\hline Overweight & 11.59 & $0.93-341.2$ & 0.156 \\
\hline Obese & 13.37 & $0.47-377.85$ & 0.128 \\
\hline Symptom duration (months) & 1.00 & $0.97-1.00$ & 0.891 \\
\hline \multicolumn{4}{|l|}{ BASDAI } \\
\hline$<4$ & Reference & & \\
\hline$\geq 4$ & 7.29 & $2.37-22.46$ & 0.001 \\
\hline \multicolumn{4}{|l|}{ Treatment } \\
\hline TNFi & Reference & & \\
\hline IL-17Ai & 1.33 & $0.48-3.66$ & 0.582 \\
\hline Steroids used & 3.98 & $1.03-15.34$ & 0.045 \\
\hline NSAIDs used ${ }^{\mathrm{a}}$ & 2.29 & $0.78-6.71$ & 0.131 \\
\hline cDMARDs used $^{\mathrm{b}}$ & 0.99 & $0.30-3.34$ & 0.992 \\
\hline
\end{tabular}

${ }^{a}$ NSAIDs used if the patient was taking any one of celecoxib, piroxicam, naproxen, ibuprofen, indomethacin, diclofenac sodium or meloxicam at index. Comparison is to those not taking NSAID

${ }^{b}$ cDMARDs used if the patient was taking any one of methotrexate, leflunomide, azathioprine, sulfasalazine, hydroxychloroquine, 6-mercaptopurrine, cyclosporine, sodium aurothiomalate, olsalazine or balsalazide at index. Comparison is to those not taking cDMARD

BASDAI, Bath Ankylosing Spondylitis Disease Activity Index; BMI, body mass index; $c D M A R D$, conventional disease modifying antirheumatic drug; NSAID, non-steroidal anti-inflammatory drug; $S D$, standard deviation

\section{Correlation between clinical signs and symptoms with sleep disturbance}

In patients administered TNFi at index, there were significant correlations between BASDAI and FACIT-Fatigue $(p<0.0001)$, BASDAI and ISI total score $(p<0.0001)$, and FACIT-Fatigue and ISI scores. In those treated with IL17Ai, only the relationship between MAPI score and BASDAI scores $(p=0.04)$ and FACIT-Fatigue and ISI scores $(p=0.0001)$ were significant (Table 4$)$. 


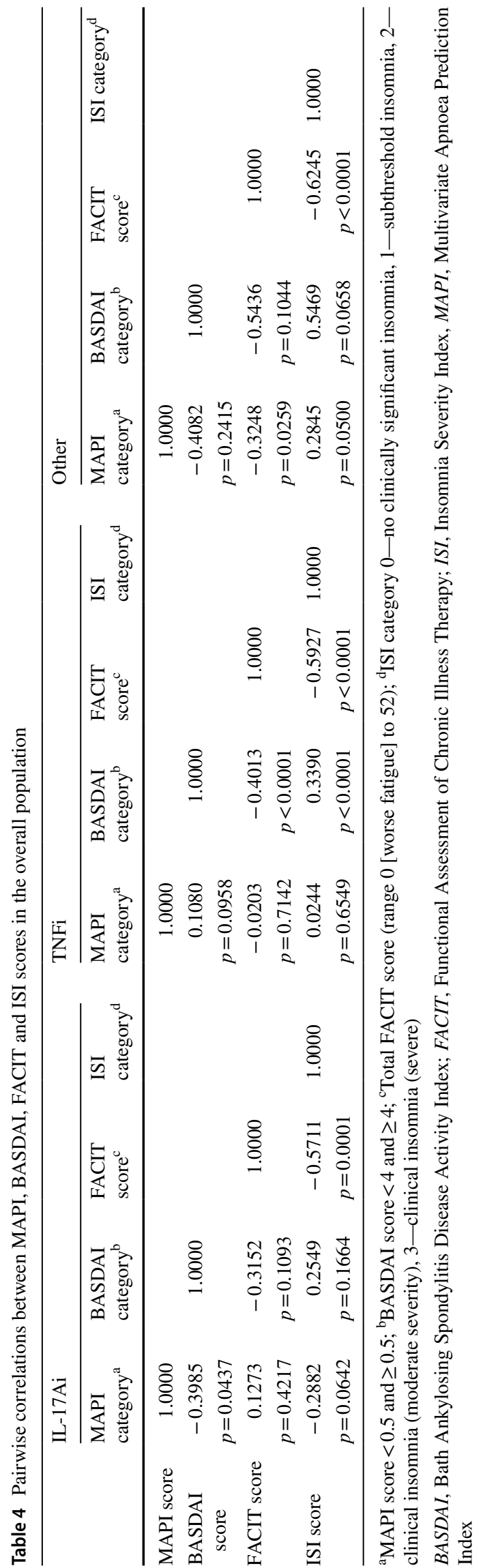

In the both IL-17Ai and TNFi populations, MAPI score was significantly negatively correlated with response to BASDAI question 6 'how long does your morning stiffness last from the time you wake up' (IL-17Ai-0.45, $p=0.02$; TNFi-0.38, $p<0.0001)$. FACIT-Fatigue score was negatively correlated with BASDAI question 4 'how would you describe the overall level of discomfort you have had from any areas tender to touch or pressure' (IL-17Ai: - 0.42, $p=0.03$; TNFi: $-0.46, p<0.0001$ ). Insomnia symptoms as measured by ISI category were significantly associated with all BASDAI questions (Table 5).

\section{Discussion}

\section{Overall findings}

In this subset of patients with AS, $19.2 \%$ reported moderate to severe clinical insomnia, and $31.5 \%$ were identified as at risk of sleep apnoea. We found no significant differences in the incidence of insomnia or sleep apnoea by treatment type in our matched population (TNFi vs IL-17Ai), with the exception of the MAPI score which was significantly higher in those treated with IL-17Ai. Those with poor disease control were more likely to experience greater sleep disturbance than those with good disease control measured by BASDAI.

\section{Implications for research and practice}

The proportions of patients who reported moderate to severe clinical insomnia or identified as at risk of sleep apnoea are within the range of previously reported estimates among AS patients [3, 4], but higher, particularly with respect to apnoea, than typical for healthy populations $[18,19]$. The high prevalence of insomnia and sleep apnoea in this realworld population of patients with AS highlights the importance of actively assessing patients in routine clinical care for signs of sleep disorders and referring patients to sleep physicians for further assessment and treatment where indicated. Disturbed sleep, including multiple nocturnal awakenings, early morning awakening, sleep onset insomnia, difficulty awakening and daytime malfunctioning can leave patients with AS feeling fatigued [20]. These sleep disorders are noted to be more severe than those experienced by patients without AS [1]. While the present study was not designed to consider the impact of commencement of treatment for sleep disorders in people with AS, treatment of sleep disorders, such as sleep apnoea, has been associated with improvements in AS [21]. Treatment of the underlying AS can also improve sleep disturbance: for example, treatment with TNFi has been reported to improve sleep disturbance in patients with AS [22], although others report that improvements are only with sleep quality, and treatment with 
Table 5 Pairwise correlation between BASDAI individual question and ISI category by treatment at index

\begin{tabular}{llllllll}
\hline & & BASDAI q1 & BASDAI q2 & BASDAI q3 & BASDAI q4 & BASDAI q5 & BASDAI q6 \\
\hline ISI category & IL-17Ai & 0.3655 & 0.3846 & 0.3477 & 0.4194 & 0.4843 & 0.4439 \\
& & $p=0.0432$ & $p=0.0327$ & $p=0.0553$ & $p=0.0188$ & $p=0.0058$ & $p=0.0124$ \\
& \multirow{2}{*}{ TNFi } & 0.4078 & 0.3616 & 0.3986 & 0.3741 & 0.3499 & 0.2617 \\
& & $p<0.0001$ & $p<0.0001$ & $p<0.0001$ & $p<0.0001$ & $p<0.0001$ & $p<0.0001$ \\
\hline
\end{tabular}

ISI category 0 - no clinically significant insomnia, 1-subthreshold insomnia, 2-clinical insomnia (moderate severity), 3-clinical insomnia (severe)

BASDAI, Bath Ankylosing Spondylitis Disease Activity Index; ISI, Insomnia Severity Index
TNFi does not improve sleep measured via polysomnography [23]. This is possibly because the specific impact of AS on sleep depends on the underlying symptomology of AS. For example, pain is associated with poor sleep efficiency (the proportion of time attempting sleep that is actually spent asleep), while elevated CRP is associated with longer sleep latency [1]. TNF $\alpha$ and IL-17A have been reported to be important in the pathogenesis of obstructive sleep apnoea; it is possible that TNF $\alpha$ is more strongly associated with its development [24, 25].

We found that those with poor disease control were more likely to experience greater sleep disturbance than those with good disease control measured by BASDAI. This may reflect lesser control of the underlying inflammatory condition (for example levels of TNF $\alpha$ and IL-17A). There is a bidirectional relationship between sleep disturbance and disease control [20]: sleep disorders may contribute to emerging pain disorders, but pain may also lead to or exacerbate sleep disorders [26]. Unsurprisingly, we found that those patients taking concomitant steroids had increased levels of sleep disturbance. This is a well characterized association [27].

We also found that men with AS were at higher risk of sleep apnoea, which reflects the known sex epidemiology of sleep apnoea [28]. Others have reported that two to three times more men without AS are diagnosed with obstructive sleep apnoea compared to women [28]. We found no association between sleep disturbances and BMI, although this was likely confounded by sex, and the relationship between BMI and severity of obstructive sleep apnoea is likely complex [28].

\section{Strengths and potential limitations}

Strengths of our study include that it is based on real-world clinical evidence collected at the point of care for patients with ankylosing spondylitis in Australia. The OPAL dataset is one of the largest datasets of patients with rheumatic diseases in the world [12]. In addition, OPAL captures multifaceted outcomes data from the patient perspective through a novel electronic patient-reported outcome (ePRO) delivery system which allows for health-related quality of life measures to be matched with clinical indices [12]. The patient-reported outcomes included in this study are clinically relevant for the initial assessment of sleep disturbance and sleep apnoea.

Our study has some potential limitations. This is a retrospective study based on data captured during routine clinical care. The analysis is therefore limited by the availability of data for secondary research in the dataset. The presence of missing data may also impact on the analysis; sample size, variables and study duration were selected to minimize the impact of missing data. Of the available patients with AS, only $9.3 \%$ were included in this study. Our assessment of sleep disorders was based on self-reported responses to sleep questionnaires and objective assessment via polysomnography, for example, was not conducted. It is possible therefore that the true estimate of sleep disorders may be lower or higher than that observed in our analysis. Additionally, the BASDAI is a subjective questionnaire, and further studies are needed to evaluate the association between sleep disturbance and objective signs of disease activity such as the ASDAS or c-reactive protein levels. Our regression modelling was hampered by poor fit, likely due to a combination of low sample sizes, and that sleep disturbance in this realworld population is likely multi-factorial and requires further investigation. For example, we did not assess the influence of exercise/physical activity on sleep as this information is not available in the dataset. Finally, we made no adjustments for multiple comparisons, and as such, it is possible that some of our statistically significant findings occurred by pure chance.

\section{Conclusions}

In this real-world cohort of patients with AS, poor disease control was associated with sleep disturbance. Little difference in sleep disturbance was observed between TNFi and IL-17Ai treatment. Screening for insomnia, sleep apnoea and fatigue in routine clinical care may provide a more holistic view of the burden of this disease, and striving to reach low disease activity may have greater positive impacts on patient quality of life beyond traditional clinical disease activity measures. 
Author contribution KT, BEB, TLS, TS, GL, RS, RT and HG were involved in the design of the study. BEB analysed the data. All authors were involved in interpreting the results of the study, the drafting or revising the manuscript, and approving it prior to submission. All authors agree to be accountable for all aspects of the work.

Funding This study was funded by Novartis Pharmaceuticals Australia Pty Ltd.

Data availability The OPAL dataset is held by OPAL Rheumatology. Data are available for research purposes upon application and approval by OPAL Rheumatology. All projects require HREC review.

Code availability Custom code in Stata MP v16.2.

\section{Declarations}

Ethics approval This study was approved by University of New South Wales (UNSW) Human Research Ethics Committee (HREC) (HC180712).

Consent to participate The activities of OPAL Rheumatology Ltd have received overarching ethics approval from the UNSW HREC, based on a patient opt-out arrangement (HC17799). This research protocol was approved by the UNSW HREC (HC180712).

Conflicts of interest/competing interests KT, TLS, GL and HG report no conflicts of interest. RT and RS are employees of Novartis Pharmaceuticals Australia Pty Ltd. TS and CO are employees of OPAL Rheumatology Ltd. BEB is an independent statistician who was contracted by OPAL Rheumatology Ltd to perform the analysis. OPAL independently developed the study design, protocol, statistical analysis plan and data analysis. We do invite the appropriate medical employee or real-world evidence team member from the sponsoring company to give input and review of the final manuscript. The decision to publish the results from this study was not dependent on the results obtained nor influenced by the sponsoring company and interpretation of the data remains with the rheumatologist/principal investigator on the study.

Open Access This article is licensed under a Creative Commons Attribution 4.0 International License, which permits use, sharing, adaptation, distribution and reproduction in any medium or format, as long as you give appropriate credit to the original author(s) and the source, provide a link to the Creative Commons licence, and indicate if changes were made. The images or other third party material in this article are included in the article's Creative Commons licence, unless indicated otherwise in a credit line to the material. If material is not included in the article's Creative Commons licence and your intended use is not permitted by statutory regulation or exceeds the permitted use, you will need to obtain permission directly from the copyright holder. To view a copy of this licence, visit http://creativecommons.org/licenses/by/4.0/.

\section{References}

1. Li Z, Fu T, Wang Y, Dong C, Shao X, Li L, Gu Z (2019) Sleep disturbances in ankylosing spondylitis: a systematic review and meta-analysis. Psychol Health Med 24(8):911-924. https://doi. org/10.1080/13548506.2019.1574357

2. Mercieca C, van der Horst-Bruinsma IE, Borg AA (2014) Pulmonary, renal and neurological comorbidities in patients with ankylosing spondylitis; implications for clinical practice. Curr Rheumatol Rep 16(8):434. https://doi.org/10.1007/ s11926-014-0434-7

3. Abdulaziez O, Asaad T (2012) Sleep problems in ankylosing spondylitis: polysomnographic pattern and disease related variables. Egypt Rheumatol 34(2):59-65. https://doi.org/10.1016/j. ejr.2012.02.001

4. Li Y, Zhang S, Zhu J, Du X, Huang F (2012) Sleep disturbances are associated with increased pain, disease activity, depression, and anxiety in ankylosing spondylitis: a case-control study. Arthritis Res Ther 14(5):R215. https://doi.org/10.1186/ar4054

5. Aydin E, Bayraktar K, Turan Y, Omurlu I, Tastaban E, Sendur OF (2015) Sleep quality in patients with ankylosing spondylitis. Rev Bras Reumatol 55(4):340-345. https://doi.org/10.1016/j.rbr.2014. 12.007

6. Fang H, Tu S, Sheng J, Shao A (2019) Depression in sleep disturbance: a review on a bidirectional relationship, mechanisms and treatment. J Cell Mol Med 23(4):2324-2332. https://doi.org/10. $1111 / \mathrm{jcmm} .14170$

7. Krueger JM (2008) The role of cytokines in sleep regulation. Curr Pharm Des 14(32):3408-3416. https://doi.org/10.2174/ 138161208786549281

8. Ming H, Tian A, Liu B, Hu Y, Liu C, Chen R, Cheng L (2019) Inflammatory cytokines tumor necrosis factor-alpha, interleukin-8 and sleep monitoring in patients with obstructive sleep apnea syndrome. Exp Ther Med 17(3):1766-1770. https://doi. org/10.3892/etm.2018.7110

9. Arias MA, Garcia-Rio F, Alonso-Fernandez A, Hernanz A, Hidalgo R, Martinez-Mateo V, Bartolome S, Rodriguez-Padial L (2008) CPAP decreases plasma levels of soluble tumour necrosis factor-alpha receptor 1 in obstructive sleep apnoea. Eur Respir J 32(4):1009-1015. https://doi.org/10.1183/09031 936.00007008

10. Walsh JA, Duffin KC, Crim J, Clegg DO (2012) Lower frequency of obstructive sleep apnea in spondyloarthritis patients taking TNF-inhibitors. J Clin Sleep Med 8(6):643-648. https://doi.org/ $10.5664 /$ jcsm. 2254

11. Roberts L, Arnold M, Bird P, Burnet S, De Jager J, Littlejohn G, Nicholls D, Tymms K, Zochling J, Griffiths H (2011) ARA-P39 optimising patient outcomes in Australian rheumatology (OPAL) - a quality use of medicines initiative (QUMI) with $>7000$ rheumatoid arthritis (RA) patients. Intern Med J 41:9-38. https://doi. org/10.1111/j.1445-5994.2010.02466.x

12. Littlejohn GO, Tymms KE, Smith T, Griffiths HT (2020) Using big data from real-world Australian rheumatology encounters to enhance clinical care and research. Clin Exp Rheumatol 38(5):874-880

13. Jones SD, Koh WH, Steiner A, Garrett SL, Calin A (1996) Fatigue in ankylosing spondylitis: its prevalence and relationship to disease activity, sleep, and other factors. J Rheumatol 23(3):487-490

14. Austin PC (2011) An introduction to propensity score methods for reducing the effects of confounding in observational studies. Multivariate Behav Res 46(3):399-424. https://doi.org/10.1080/ 00273171.2011 .568786

15. Shahid A, Wilkinson K, Marcu S, Shapiro CM (2011) Insomnia severity index (ISI). In: Shahid A (ed) STOP, THAT and one hundred other sleep scales. pp 191-193. https://doi.org/10.1007/ 978-1-4419-9893-4 43

16. Brandt J, Haibel H, Cornely D, Golder W, Gonzalez J, Reddig J, Thriene W, Sieper J, Braun J (2000) Successful treatment of active ankylosing spondylitis with the anti-tumor necrosis factor alpha monoclonal antibody infliximab. Arthritis Rheum 
43(6):1346-1352. https://doi.org/10.1002/1529-0131(200006) 43:6<1346::AID-ANR18>3.0.CO;2-E

17. Maislin G, Pack AI, Kribbs NB, Smith PL, Schwartz AR, Kline LR, Schwab RJ, Dinges DF (1995) A survey screen for prediction of apnea. Sleep 18(3):158-166. https://doi.org/10.1093/sleep/ 18.3 .158

18. Adams R, Appleton S, Taylor A, Mcevoy D, Antic N (2016) Report to the Sleep Health Foundation. 2016 sleep health survey of Australian adults

19. Adams RJ, Appleton SL, Taylor AW, Gill TK, Lang C, McEvoy RD, Antic NA (2017) Sleep health of Australian adults in 2016: results of the 2016 Sleep Health Foundation national survey. Sleep Health 3(1):35-42. https://doi.org/10.1016/j.sleh.2016.11.005

20. Tymms K (2019) Impact of sleep disturbance in ankylosing spondylitis. Research Reviews

21. Wang Y, Lin S, Li C, Shi Y, Guan W (2020) Sleep apnea-hypopnea syndrome caused by ankylosing spondylitis: a case report. Medicine (Baltimore) 99(19):e20055. https://doi.org/10.1097/ MD.0000000000020055

22. Karadag O, Nakas D, Kalyoncu U, Akdogan A, Kiraz S, Ertenli I (2012) Effect of anti-TNF treatment on sleep problems in ankylosing spondylitis. Rheumatol Int 32(7):1909-1913. https://doi.org/ 10.1007/s00296-011-1907-x

23. Karatas G, Bal A, Yuceege M, Firat H, Gurcay E, Ardic S, Cakci FA (2018) Evaluation of sleep quality in patients with ankylosing spondylitis and efficacy of anti-TNF-alpha therapy on sleep problems: a polisomnographic study. Int J Rheum Dis 21(6):12631269. https://doi.org/10.1111/1756-185X.13102
24. Ying L, Li H, Pan Z, Ma S, Zhang P, Wang Q, Lu G, Zhou J (2014) Relationship of redundant Th17 cells and IL-17A, but not IL-17 F, with the severity of obstructive sleep apnoea/hypopnoea syndrome (OSAHS). BMC Pulm Med 14:84. https://doi.org/10. 1186/1471-2466-14-84

25. Krueger JM, Obal FJ, Fang J, Kubota T, Taishi P (2001) The role of cytokines in physiological sleep regulation. Ann N Y Acad Sci 933:211-221. https://doi.org/10.1111/j.1749-6632.2001.tb058 26.x

26. Yolbas S, Yildirim A, Duzenci D, Gundogdu B, Ozgen M, Koca SS (2017) Sleep quality, sleeping postures, and sleeping equipment in patients with ankylosing spondylitis. Turk J Med Sci 47(4):1198-1205. https://doi.org/10.3906/sag-1605-62

27. McDonough AK, Curtis JR, Saag KG (2008) The epidemiology of glucocorticoid-associated adverse events. Curr Opin Rheumatol 20(2):131-137. https://doi.org/10.1097/BOR.0b013e3282f51031

28. Jordan AS, McEvoy RD (2003) Gender differences in sleep apnea: epidemiology, clinical presentation and pathogenic mechanisms. Sleep Med Rev 7(5):377-389. https://doi.org/10.1053/smrv.2002. 0260

Publisher's note Springer Nature remains neutral with regard to jurisdictional claims in published maps and institutional affiliations. 